International Journal of Advanced Trends in Computer Science and Engineering

Available Online at http://www.warse.org/IJATCSE/static/pdf/file/ijatcse60852019.pdf

https://doi.org/10.30534/ijatcse/2019/60852019

\title{
Composite Radioisotope Coating Parameters and Reflecting Characteristics Calculation Selection Method
}

\author{
Savchenko Vitalii ${ }^{1}$, Zaika Viktor ${ }^{2}$, Trembovetskyi Maksym ${ }^{3}$, Shuklin German ${ }^{4}$, Berkman Liubov ${ }^{5}$, \\ Storchak Kamila ${ }^{6}$, Rolin Ihor $^{7}$ \\ ${ }^{1}$ Doctor of Technical Sciences, Professor, Director of the Information Security Institute, State University of \\ Telecommunications, Ukraine, savitan@ukr.net \\ ${ }^{2}$ Doctor of Technical Sciences, Associated Professor, Head of the Department of Telecommunications and \\ Networks, State University of Telecommunications, vfzaika@mail.ru \\ ${ }^{3}$ Doctor of Technical Sciences, Senior Research Associate, Head of the Department of Energy Efficient \\ Technologies, State University of Telecommunications, maksimtremboff@gmail.com \\ ${ }^{4}$ Philosophy Doctor, Head of the Department of Information Security Technical Systems, State University of \\ Telecommunications, mathacadem-kiev@ukr.net \\ ${ }^{5}$ Doctor of Technical Sciences, Professor, Deputy Head of the State University of Telecommunications, State \\ University of Telecommunications, Ukraine, dutzv@ukr.net \\ ${ }^{6}$ Doctor of Technical Sciences, Head of Information Systems and Technologies Department, State University of \\ Telecommunications, Ukraine, kpstorchak@ukr.net \\ ${ }^{7}$ Doctor of Technical Sciences, Professor of the department of the tactics and special subjects of the Militari \\ Institute of the Tenk Troops of the Nationnal Technical University "Kharkiv Polytechnic Institute"
}

\begin{abstract}
The development results of a selection method for the composite radioisotope coatings (CRC) parameters and determining their reflective properties in a wide frequency range are presented. Parameters necessary for calculating reflective properties are indicated. The basic design relationships for evaluating the reflective properties of the objects with a large-scale distribution are given.

The procedure for calculating the real and imaginary parts of the semiconductor dielectric constant coating layer is shown. The numerical values of the average reflection coefficient from the CRC scattering are given, as well as possible variants of the coating structure.
\end{abstract}

Key words: composite radioisotope coatings, dielectric constant, reflective properties, coating structure.

\section{INTRODUCTION}

Studies in the area of creating broadband radar absorbing coatings that have the small mass and the size characteristics have shown the promise of the CRC [1-6]. The basis of these coatings are the III - V group semiconductors with radioisotope inclusions, which are pure sources of $\alpha$ particles: polonium 210 (Po-210), strontium-90 (St-90), and plutonium-238 (Pu-238). Most preferred are St-90 and $\mathrm{Pu}-238$ due to their lower biological hazard. The use of radioisotope inclusions introduces a number of qualitative additions to the composite material in comparison with conventional composite materials. The most important ones leading to a significant change in the dielectric and related electrodynamics properties of the coating are:
- the non equilibrium states sources emergence of the material electronic subsystem and the surrounding air environment;

- unsteadiness of the material radio physical properties due to a constant change in the conductivity internal structure due to changes in the number of tracks from the radioisotope inclusions decay products $[1,2,5]$.

In the works of A. Sotnikov, O. Vorobyev etc. [1-3, 6-7], it was shown that the main CRC advantage along with the expansion of the pass band is the simultaneous combination of all the basic properties inherent in the coatings known types separately [2, 3]. That is, the advantages of the CRC are due to the ability to provide the required reflective, absorbing and radioactive characteristics in an extended frequency range by choosing the size, shape, type of inclusions material, structure and distribution of spatial homogeneity. The implementation completeness of these advantages in practice is based on the following factors [3, 4]:

1) the effects on the absorption and reflection of electromagnetic waves (EMW) incident on the coating, the electronic subsystem non equilibrium states of the weakly ionized air medium due to the radioisotope inclusions;

2) the absorbing properties influence of the non equilibrium states of the electronic subsystem material due to the radioisotope inclusions;

3) the effects on the EMW scattering of the tracks unsteadiness arising due to $\alpha$-particles emitted by radioisotope inclusions;

4) the influence on the EMW attenuation of the coating structure heterogeneous and irregular conductivity; 
5) the influence of the material nonlinear properties on the conversion of the detection means signals.

The non equilibrium state in a coating with radioisotope inclusions can be interpreted as in homogeneity in the momentum space limited by the dimensions of the coating and the airborne weakly ionized medium adjacent to it.

The irregular distribution of the CRC radioisotope inclusions, as well as the unsteady conductive inclusions structure at a concentration of inclusions at the percolation threshold, leads to the abnormally high EMW.

\subsection{Problem analysis}

The theoretical studies results conducted by the authors in the field (area)of creating effective radar absorbing coatings indicate the promise of the CRC creation and application in many areas related to solving problems of reducing the secondary radiation level of objects. At the same time, the practical implementation of the proposed physical mechanisms requires knowledge of the corresponding CRC parameters, at which the necessary level of secondary radiation will be provided. In addition, it is necessary to have an appropriate coating structure and determine the basic design relationships for evaluating the reflective properties of objects with a large-scale distribution. The solution of these problems necessitates the development of an appropriate methodology for choosing the composite radioisotope coatings parameters and calculating reflective characteristics.

The aim of the article is to develop a methodology for choosing the composite radioisotope coatings parameters and to evaluate their reflective properties in a wide frequency range.

\section{MAIN MATERIAL}

\subsection{The main parameters coating definition}

To perform the CRC reflective properties objects calculations and the basic requirements presentation for the coatings characteristics at the stage of their design, we list the main parameters that determine their reflective and absorbing properties. These parameters include:

1. Radioactive inclusions activity $-\kappa_{\operatorname{rad}}[\mathrm{Ku}]$;

2. The $\alpha$ initial energy - particles $-\mathrm{E}_{\alpha}[\mathrm{MeV}]$;

3. The average inclusions radius $-r_{m}[\mu \mathrm{m}]$;

4. The track $\alpha$ average radius - particles $-r_{t r}[\mu \mathrm{m}]$;

5. Coating matrix material $-\varepsilon_{\mathrm{m}}$;

6. The mean $\alpha$ free path- particles in the matrix $-1_{\operatorname{tr}}$ $[\mu \mathrm{m}]$;

7. The coating characteristic resonant frequencies $-\omega_{\text {ri }}$ [GHz];

8. Atmospheric pressure $-\mathrm{p}_{\mathrm{V}}[\mathrm{atm}]$, at which the coating will be applied;
9. The mean $\alpha$ free path- particles in the air $-1_{\mathrm{V}}\left(\mathrm{p}_{\mathrm{V}}\right)$ [cm];

10. The semiconductor thickness layer of the composite material - $\mathrm{h}[\mathrm{mm}]$;

11. The semiconductor conductivity layer of the composite material $-\sigma_{\mathrm{m}}[\mathrm{S}]$;

12. The inclusion conductivity material $-\sigma_{\mathrm{d}}[\mathrm{S}]$;

13. The matrix materials and the inclusion density $-\rho_{m}$

и $\rho_{\mathrm{d}}\left[\mathrm{g} / \mathrm{cm}^{3}\right]$;

14. The matrix radioisotope inclusions concentration $\mathrm{c}_{\mathrm{d}} 1 / \mathrm{cm}^{3}$.

When substantiating the coatings general requirements, an important role is played by the overall dimensions requirements of the coating, which, as a rule, should be of negligible size and mass and provide the reflection required electromagnetic radiation characteristics in a

given frequency range $\omega_{\min }<\omega<\omega_{\max }$.

Since the concentration of radioisotope inclusions in the semiconductor layer, determined by the condition of the significant non linearity appearance for surface currents in the coating, is selected at the percolation threshold $\mathrm{c}_{\mathrm{m}} \approx 0.5$, the average coating density will be determined in accordance with the expression [8]:

$$
\rho_{\mathrm{av}}=\rho_{\mathrm{m}}\left(1-\mathrm{c}_{\mathrm{d}}\right)+\rho_{\mathrm{d}} \mathrm{c}_{\mathrm{d}} .
$$

The requirements for mass $(\mathrm{M})$ and coating area $(\mathrm{S})$ naturally determine its thickness $h=\frac{\mathrm{M}}{\rho_{\mathrm{av}} \mathrm{S}}$.

\subsection{Basic design ratios for evaluating the CRC reflective properties objects}

The reflection properties can be calculated using the example of a two-layer composite radioisotope coating with the surrounding air ionization.

The CRC dielectric constant for the normal EMW dip case along the axis $\mathrm{Z}$ can be represented as follows:

$$
\begin{aligned}
& \varepsilon(\omega, \mathrm{k})=\left(1-\frac{4 \pi \mathrm{n}_{\mathrm{e}_{\mathrm{ns}}} \mathrm{e}^{2}}{\mathrm{~m}_{\mathrm{e}} \omega^{2}}\right)(1-0,5 \mathrm{i}) \theta\left(1_{\mathrm{v}}-\mathrm{z}\right) \theta(\mathrm{z})+ \\
& +\theta(-\mathrm{z}) \theta(\mathrm{z}+\mathrm{h}) \times\left(\begin{array}{c}
\varepsilon_{\mathrm{eff}}\left(\mathrm{D}_{\mathrm{f}}, \omega_{\mathrm{ri}}, \omega\right) \xi(\mathrm{t}) \times \\
\times\left(1-\mathrm{i}\left(0,5+v_{\text {eff }}\left(\mathrm{D}_{\mathrm{f}}, \omega\right)\right)\right)
\end{array}\right),
\end{aligned}
$$

where $\theta(t)$ - is the Heaviside function; 
$\xi(t)$ - a time function that describes the non-stationary coating properties and is determined by the non-stationary impedance;

$\omega_{r_{1}}-$ a set of the composite material resonant frequencies with radioisotope inclusions;

$v_{\text {eff }}\left(D_{f}, \omega\right)$ - the magnitude of the electromagnetic radiation attenuation at a frequency $\omega$, depending on the non equilibrium state of the electronic subsystem a semiconductor layer in accordance with the nature of the radioisotope distribution inclusions;

$\varepsilon_{\mathrm{m}}\left(\mathrm{D}_{\mathrm{f}}, \omega_{\mathrm{ri}}, \omega\right)$ - the semiconductor layer dielectric constant, depending on the inclusions and their resonant frequencies material.

In expression (2), the first term is determined by the non equilibrium state of the weakly ionized air medium arising under the radioisotope inclusions influence, the second term is determined by the inclusions dielectric properties in the semiconductor layer, as well as by the non equilibrium state of the material electronic subsystem along the $\alpha$ particles tracks.

The degree of the resulting air surface layer ionization is determined by the radioisotope inclusions activity and their surface density in accordance with the expression:

$$
\kappa_{\mathrm{S}} \approx \kappa_{\mathrm{rad}} / \mathrm{S}_{\mathrm{eff}}
$$

Due to ionization in a volume of $1 \mathrm{~cm}^{3}$ per 1s electrons are formed $\mathrm{P}_{\mathrm{e}}=3,7 \cdot 10^{10} \kappa_{\mathrm{s}} \frac{\mathrm{E}_{\alpha}}{\mathrm{I}_{\mathrm{i}} 1_{\text {tr }}}$. The $\alpha-$ particles energy arising from nuclear decay does not change very much and lies in the region of several $\mathrm{MeV}$. In further estimates, we will consider this energy to be of the $\mathrm{E}_{\alpha} \approx 5 \mathrm{MeV}$ order.

The mean $\alpha^{-}$particles free path in the air is about $3.8 \mathrm{~mm}$ at a pressure of $1 \mathrm{~atm}$ [9]. Depending on the object height with the protective coating in question, the ionized nearsurface layer different thicknesses will take place.

In a weakly ionized air medium, the incident electromagnetic wave energy is absorbed, and the open space impedance $Z_{V}(\kappa)$ is coordinated with the surface impedance of the CRC semiconductor layer $Z_{m}$. The coordination impedance leads to a significant reflection weakening from the coating surface:

$$
R_{v}(\kappa)=\frac{Z_{m}-Z_{v}(\kappa)}{Z_{m}+Z_{v}(\kappa)},
$$

where $\kappa=\frac{2 \pi}{\lambda}-$ is the wave number.

In accordance with expression (4), the surface (R) reflection coefficient is reduced by the amount that can be determined from the relation (ratio):

$$
\delta \mathrm{R}(\kappa)=\frac{\delta \varepsilon_{\mathrm{nq}}(\kappa)}{\sqrt{\varepsilon_{\mathrm{m}}}},
$$

where $\delta \varepsilon_{\mathrm{nq}}(\kappa)$ - where is the non equilibrium state contribution of the weakly ionized air medium electronic subsystem to the electromagnetic radiation absorption.

The non equilibrium state influence of the CRC electronic subsystem on the dielectric constant can be estimated on the basis of the following relation $[5,8]$ :

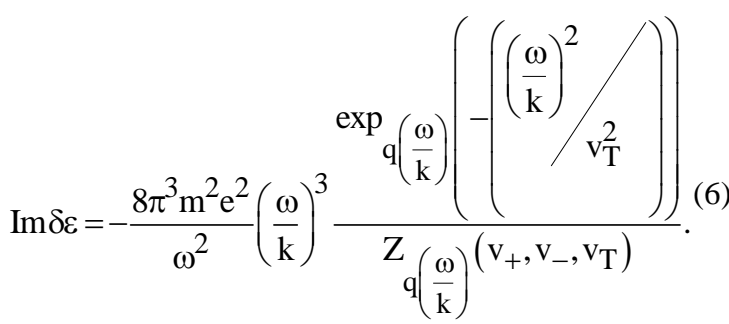

The signal attenuation at a wavelength $\lambda$ in an airborne surface of a slightly ionized medium can be written in the following form:

$$
\mathrm{R}_{\text {eff }_{\mathrm{v}}}(\kappa, \lambda)=\mathrm{R}_{\mathrm{v}}(\kappa) \exp \left(-2 v_{\operatorname{eff}_{\mathrm{v}}} \frac{1_{\mathrm{v}}(\kappa)}{\lambda}\right)
$$

where $\quad v_{\text {eff }}{ }_{v}-$ is the effective absorption of electromagnetic radiation in the weakly ionized air layer.

The EMW passing through the ionized air layer to the coating surface enters the semiconductor layer with the non equilibrium state of its electronic subsystem.

The mean $\alpha$-particles free path with the energy $E_{\alpha}$ $(\mathrm{MeV})$ in a semiconductor material is determined in accordance with the expression [10]:

$$
1_{\mathrm{tr}} \approx 1,741 \cdot 10^{-4} \frac{\mathrm{A}}{\rho_{\mathrm{av}}} \frac{\mathrm{m}_{\mathrm{e}}}{\mathrm{M}_{\mathrm{a}}}\left(\frac{\mathrm{M}_{\mathrm{a}}}{\mathrm{m}_{\mathrm{e}}} \mathrm{E}_{\alpha}\right)^{3 / 2},
$$

where $\mathrm{A}$ - is the atomic weight of the semiconductor coating layer material.

The track radius of an $\alpha$ particle in a composite material can be considered known, since it does not change significantly with changing parameters in the wavelength range of interest to us and is of the $\mathrm{r}_{\mathrm{tr}} \approx 10^{-6} \mathrm{~cm}$ order. 
The semiconductor layer with the inclusions has a large frequencies spectrum, the values of which determine the broadband coating degree.

Consider the main resonant frequencies.

Resonance frequencies associated with the average distance between the tracks $\Delta \mathrm{l}_{\mathrm{tr}}$. We estimate these distances through surface activity and relaxation time. Then $\Delta \mathrm{l}_{\mathrm{tr}} \approx\left(\kappa_{\mathrm{s}} \tau_{\mathrm{i}}\right)^{-1 / 2}$ or, measuring activity in micro curie, relaxation time in milliseconds, we get in centimeters:

$$
\Delta \tilde{\mathrm{l}}_{\mathrm{tr}} \approx 0,3 \frac{1}{\sqrt{\kappa_{\mathrm{s}} \tau_{\mathrm{i}}}} .
$$

For the $0,1 \mathrm{mc}$ order relaxation time and the order of $50 \mu \mathrm{Ku} / \mathrm{cm}^{2}$ activity, this formula yields the average resonance frequency value of the order of:

$$
\tilde{\omega}_{\mathrm{r}_{1}} \approx \frac{\mathrm{c}}{0,3} \sqrt{\kappa_{\mathrm{s}} \tau_{\mathrm{i}}} \approx 2 \dot{0} \mathrm{GHz}
$$

The radioisotope inclusions dimensions provide a resonant frequency $\omega_{\mathrm{r}_{2}}$ in the terahertz range.

The remaining frequencies $\omega_{\mathrm{r}_{1}}$ are much lower and are determined by the average distances between inclusions and the semiconductor coating layer thickness.

The effective signal attenuation at a wavelength $\lambda$ in the semiconductor coating layer can be written as follows:

$$
\mathrm{R}_{\mathrm{eff}}(\kappa, \lambda)=\mathrm{R}_{\Pi}(\kappa) \exp \left(-2 v_{\operatorname{eff}} \frac{\mathrm{l}_{\mathrm{tr}}}{\lambda}\right)
$$

It should be considered that the electromagnetic radiation attenuation magnitude $v_{\mathrm{eff}}$ is determined by the non equilibrium state of the semiconductor coating layer electronic subsystem, to create which it is necessary to use radioisotope inclusions with the order $70 \mu \mathrm{Ku} / \mathrm{cm}^{2}$ activity [3].

The signal reflected from the $\alpha$ particles tracks and radioisotope inclusions, taking into account the attenuation in a non equilibrium medium, excites the surface conduction currents, which form the secondary radiation.

The secondary radiation structure is determined by the coating structure: the complex conductivity spatial structure and its unsteadiness, which leads to an increase in the effective energy loss in an in homogeneous unsteady medium even at sufficiently long waves.

The radioactive inclusions presence in the coating determines the structure significant unsteadiness within the above mentioned time of the means signals accumulation for the secondary radiation recording.
When certain requirements for the coating parameters ( $\left.\mathrm{r}_{\mathrm{d}}=0,05 \mathrm{~mm}\right)$, are met, strong fluctuations in the reflection of electromagnetic waves in time arise.

Such stochastic bursts lead to a decrease in the amplitude of the reflected signal during bursts (up to $25 \mathrm{~dB}$ ) and a significant expansion of the secondary radiation frequency range. The frequency range extension leads to a significant decrease in the flux density of the reflected power in a given frequency band.

\subsection{The material calculating example and imaginary parts of the semiconductor coating layer dielectric constant}

As the material of the coating solid-state part, we choose a semiconductor of the group AIIIBV - indium phosphate InP, whose high-frequency permeability is equal $\varepsilon_{\mathrm{m}}=15,68$.

Taking into account the radioisotope inclusions, the dielectric constant of which is chosen to be $\varepsilon_{\mathrm{d}}=4,5+\mathrm{i} 0,2$, the dielectric constant of the semiconductor coating layer effective value with inclusions can be determined by the formula [5]:

$$
\varepsilon_{\text {eff }} \mathrm{m}=\varepsilon_{\mathrm{d}} \frac{1+2 \mathrm{c}_{\mathrm{m}} \frac{\varepsilon_{\mathrm{m}}-\varepsilon_{\mathrm{d}}}{2 \varepsilon_{\mathrm{d}}+\varepsilon_{\mathrm{m}}}}{1-\mathrm{c}_{\mathrm{m}} \frac{\varepsilon_{\mathrm{m}}-\varepsilon_{\mathrm{d}}}{2 \varepsilon_{\mathrm{d}}+\varepsilon_{\mathrm{m}}}}
$$

Substituting the above parameters into expression (11), we obtain $\varepsilon_{\text {eff }}=8,7$.

In accordance with the selected parameters of the coating material, numerical calculations of its real frequency dependence and imaginary parts of the dielectric constant were performed taking into account the non equilibrium state of the electronic subsystem. The calculation results are presented in the form of graphs in Figure. 1 and 2.

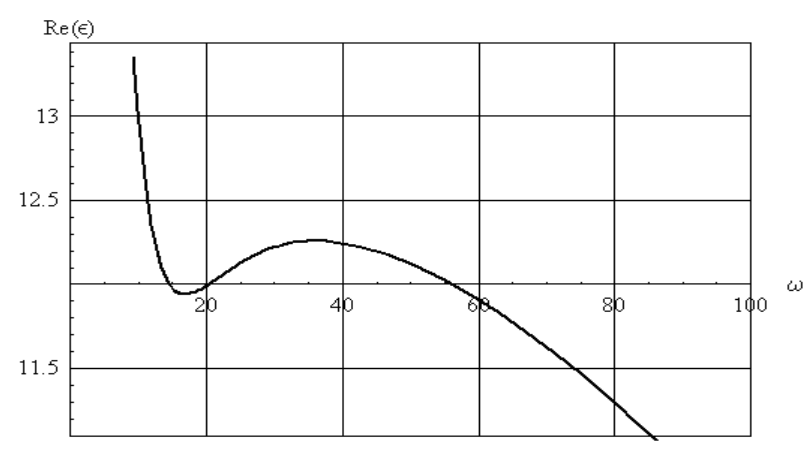

Figure 1: Dependence of the semiconductor coating layer dielectric constant real part average value on the frequency The dependence calculating results of the CRC reflection coefficient on the frequency for three values of the layer thickness $(\mathrm{h}=1 \mathrm{~mm}, 2 \mathrm{~mm}, 0,5 \mathrm{~mm})$ are shown in Fig. 2, 3. 


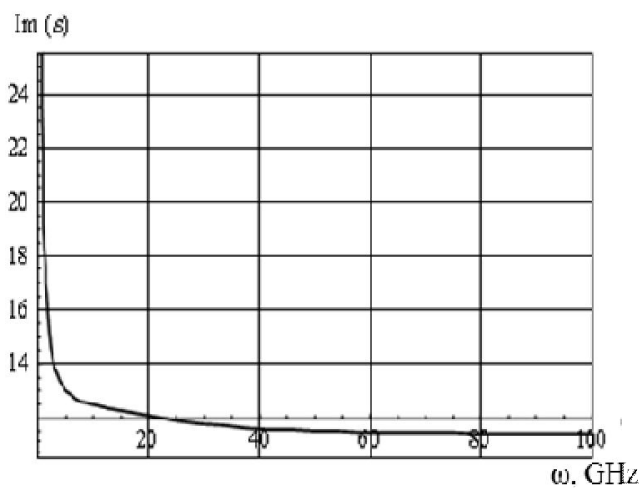

Figure 2 : The average value dependence of the semiconductor coating layer dielectric constant imaginary part on the frequency

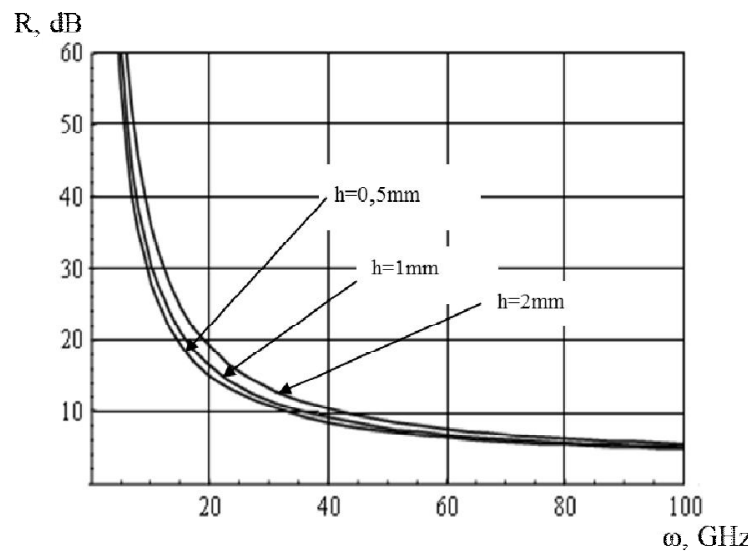

Figure 3:- The dependence of the EMW CRC reflection coefficient average value on the frequency

The graphs analysis shown in Figure 2 and 3 shows that the real and imaginary parts values of the semiconductor coating layer dielectric constant with radioisotope inclusions are of the same order in a wide frequency band. This provides a reflection coefficient of the same order in a wide frequency band.

Thus, a technique to choose the CRC parameters and evaluate its reflective properties is proposed.

To expand the coating frequency range (for example, in the direction of increasing the operating frequency), it is necessary to use radioisotope inclusions with greater activity. The thickness (h) semiconductor coating material with different radioisotope inclusions activity values can be considered by this parameter as a set the coating material separate layer. In this case, the CRC reflection coefficient determination scattering will be carried out as if for a multi layer structure.

The study results of the absorption main physical mechanisms and scattering incident on the EMW composite radioisotope coating make it possible to develop a number of coating structures for application to real objects, taking into account the peculiarities of their use and operation.

Obviously, the differences in the requirements for the reflective properties of ground and air objects determine the differences in the coating construction principles, associated with the use of a different physical mechanisms set for the absorption and scattering of electromagnetic waves, which ensure the required objects reflective properties.

\subsection{Features of the CRC air objects construction}

Satisfying stringent requirements, for example, to reduce the radar visibility of airborne objects, depending on the object type in terms of habitability, causes a certain difference in the coating construction principles. Coatings applied to aircraft should not use the EMW absorption mechanisms associated with the ionization of airspace adjacent to an object. Therefore, the most effective CRC structure for application to inhabited airborne objects will be a structure in which the absorption and scattering mechanisms of electromagnetic waves arising in the semiconductor layer are used.

In this case, the coating structure will have the form shown in Figure 4.

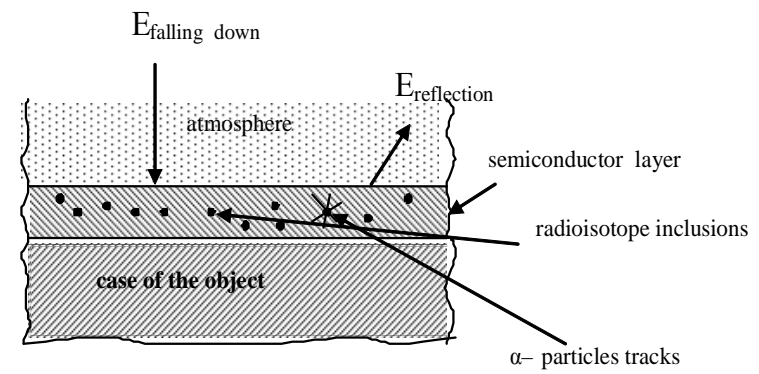

Figure 4: The coating structure

For this CRC structure, the dielectric constant in accordance with the expression (2) will be determined by the following expression:

$$
\begin{aligned}
& \varepsilon(\omega, \mathrm{k})=\varepsilon_{\mathrm{m}}\left(\mathrm{D}_{\mathrm{f}}, \omega_{\mathrm{ri}}, \omega\right) \xi(\mathrm{t}) \times \\
& \times\left(1-\mathrm{i}\left(0,5+v_{\text {eff }}\left(\mathrm{D}_{\mathrm{f}}, \omega\right)\right)\right)
\end{aligned}
$$

To protect the ground equipment, the most effective coating structure model is shown in Figure. 5.

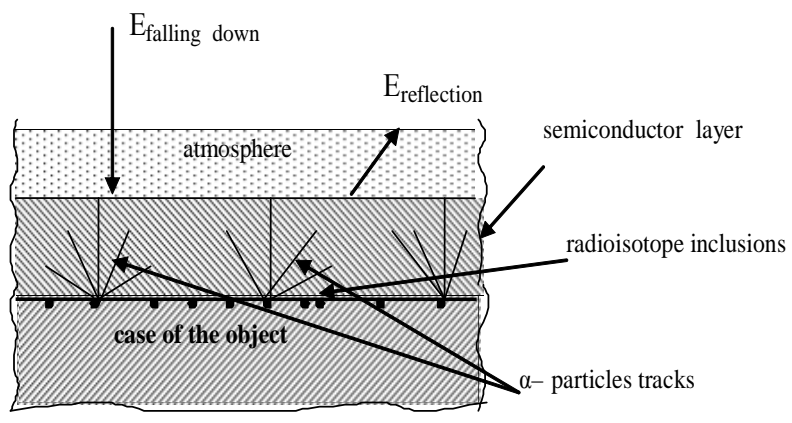

Figure 5: The coating structure, providing a decrease in the objects reflective properties due to the scattering of electromagnetic waves on the unsteady structure of tracks

- particles and absorption due to the emerging non equilibrium states of the material electronic subsystem along the tracks. 


\section{CONCLUSION}

A technique has been developed for choosing the CRC multi layer parameters, which consists of taking into account the reflective properties physical mechanisms of reducing the material based on the radiation exposure to dielectric materials.

It is shown that the real and imaginary parts values parts of the semiconductor coating layer dielectric constant with radioisotope inclusions are of the same order in a wide frequency band. This provides a reflection coefficient of the same order in a wide frequency band.

Coating structures are proposed that implement a different set of physical mechanisms taking into account radiation safety requirements.

Using the selection recommendations of the main CRC parameters will provide the required values of reflective and absorbing and properties of coatings in a wide frequency range.

\section{REFERENCES}

1. A.V. Krizny, O.M. Vorobyov, and O.M. Sotnikov. Designing the structure of the material of the protective screens of radio-electronic means of arms and military equipment from the effects of powerful electromagnetic radiation pulse duration. Trudy Universitetu, 6 (120), 2013, pp. 187-191.

2. O. Vorobiov, V. Savchenko, A. Sotnikov, V. Tarshin, and T. Kurtseitov. Development of radioisotopic-plasmatechnology for the protection of radio electronic means from powerful electromagnetic radiation. Eastern-European Journal of Enterprise Technologies, 5(85), 2017, pp. 16-22. doi:10.15587/1729-4061.2017.91642.

3. A. Sotnikov, M. Iasechko. Counteraction to the powerful electromagnetic radiation is for defence of radio electronic facilities. Information processing systems, 9(135), Kharkiv, Ukraine, 2017, pp. 76-81.

4. M. Iasechko. Plasma technologies for the protection of radio electronic means from exposure to high-power electromagnetic radiations with ultrashort pulse duration, Proceedings of the 1-st Annual Conference, Tallinn, Estonia, 2017, pp. 18-21. doi: /10.21303/2585-6847.2017.00480.

5. M. Iasechko, V. Larin, S. Salkutsan, L. Mikhailova, O. Kozak, O. Ochkurenko Formalized model descriptions of modified solid-state plasma-like materials to protect radio-electronic means from the effects of

electromagnetic radiation, IJATCSE, 8(3), 2019, pp. 300304. doi:/10.30534/ijatcse/2019/09832019.

6. A. Sotnikov, M. Iasechko. Counteraction to the powerful electromagnetic radiation is for defence of radio electronic facilities. Information processing systems, 9(135), Kharkiv, Ukraine, 2017, pp. 76-81.

7. M. Iasechko, A.Sotnikov, V. Tarshyn. Impact of powerful electromagnetic radiation on radioelectronic means, International Journal of Advanced Trends in Computer Science and Engineering, 8(3), May - June 2019, 393 - 398398 Air Forces of the
Armed Forces of Ukraine, 3, Kharkiv, Ukraine, 2017, pp. 86-91.

https://doi.org/10.30748/nitps.2017.28.11

8. A. Sotnikov, M. Iasechko The use of plasma technology to protect radio electronic equipment from exposure to electromagnetic radiation, Vestnik NTU "KhPI", Series: New solutions in modern technologies, 53(1274), Kharkiv, Ukraine, 2017, pp. 182-187. doi:10.20998/2413-4295.2017.53.25.

9. A. Sotnikov, M. Iasechko Advanced technologies of radio electronic equipment (means) protection from powerful electromagnetic radiations with ultra short duration of pulses exposure, Published by Izdevnieciba Baltija Publishing, Collective monograph, Riga, 2018, pp.356-385.

10. O. G. Sytenko. Electromagnetic plasma fluctuations, $K h G P U$, Ukraine, Kharkiv, 1965, pp. 1183. 